\title{
Algebraic description of spacetime foam
}

\author{
Ioannis Raptis*and Roman R. Zapatrin ${ }^{\dagger}$
}

11 July 2002

\begin{abstract}
A mathematical formalism for treating spacetime topology as a quantum observable is provided. We describe spacetime foam entirely in algebraic terms. To implement the correspondence principle we express the classical spacetime manifold of general relativity and the commutative coordinates of its events by means of appropriate limit constructions.
\end{abstract}

\section{Physical Motivation}

In this paper we present an algebraic model of spacetime foam. The notion of spacetime foam has manifold and somewhat ambiguous meaning in the literature if only because the models vary. There is no unanimous agreement about what foam 'really' pertains to mainly due to the fact that each of the mathematical models highlights different aspects of that concept. Here we use the term 'foam' along the concrete but general lines originally introduced by Wheeler [43] who intended to refer to a spacetime with a dynamically variable, because quantally fluctuating, topology.

The basic intuition is that at quantum scales even the topology of spacetime is subject to dynamics and interference. This conception of foam is in glaring contrast with general relativity, the classical theory of gravity, where spacetime is fixed to a topological manifold once and forever so that the sole dynamical variable is a higher level structure, namely, the spacetime geometry. It seems theoretically lame and rather ad hoc to regard the geometry of spacetime as being a dynamical variable attribute that can in principle be measured (ie, an observable property of spacetime), while at the same time to think of its topology as a structure a priori fixed by the theoretician, an

*EU Marie Curie Postdoctoral Research Fellow, Theoretical Physics Group, Blackett Laboratory, Imperial College of Science, Technology and Medicine, Prince Consort Road, South Kensington, London SW7 2BZ, UK; e-mail: i.raptis@ic.ac.uk

${ }^{\dagger}$ Quantum Information Group, ISI, Villa Gualino, V.le S.Severo 65, 10133, Torino, Italy; e-mail: zapatrin@isiosf.isi.it (address for correspondence) 
inert ether-like absolute background that is not liable to experimental investigation thus effectively an unobservable theoretical entity [10]. Especially in the quantum realm where everything seems to be in a state of dynamical flux and quantum superposition, it appears to be quite plausible that not only the metric but also the spacetime topology manifests such a 'standard' quantum behaviour. Hence, the theoretical investigation of spacetime foam as conceived above appears to be part and parcel of our apparently never ending quest for a cogent quantum theory of spacetime structure and its dynamics (ie, quantum gravity proper).

So, we will treat the topology of spacetime as a quantum observable, that is, as a measurable dynamical property of spacetime that engages into coherent quantum superpositions. In keeping with the general operationalist approach to quantum theory as originally championed by Heisenberg, we give an entirely algebraic formulation of spacetime foam. As befits the innate reticularity of quantum systems, our algebraic models are combinatorial structures of strongly finitistic character. Indeed, such socalled locally finite or 'finitary' models seem to be suitably designed to undermine the non-operational and unphysical nature of the classical spacetime continuum of general relativity. Laying the non-dynamical character of the manifold model for spacetime as described in the opening paragraph aside for a moment, its gravely non-operational trait is mainly that we have no actual experience of a continuous infinity of events since we always seem to record a finite number of them in laboratories of finite size during experiments of finite duration. At the same time, its characterisation above as being unphysical pertains to that we can in principle pack an uncountable infinity of events in a finite spacetime volume which is likely to be the main culprit for the unrenormalisable infinities that plague a quantum field theoresis of gravity on a spacetime manifold. Moreover, in an algebraic modelling of such discrete combinatorial spacetimes, one naturally expects that the representations of the algebras involved will also be finite dimensional if only to abide to the principle of finiteness as much as possible throughout the construction of the theory. All in all, it seems appropriate to call such a finitary algebraic approach to dynamical quantum spacetime topology or foam 'pragmatic' 31.

Of course, if the theory is to qualify as being physically plausible, realistic and literally pragmatic, then the notion of topology, commonly understood as the mathematical theory of the properties of space proper, seems an inappropriate starting point for the unification of relativity and the quantum. From a relativistic point of view 'spatiality' or 'spacelikeness' is fundamentally an unphysical conception of the connections between events since we have no experience of acausal or tachyonic dynamical propagations at least as regards our experimental familiarity with matter quantum field actions which seem to obey the principle of Einstein causality ( $i e$, that acausally connected or spacelike separated matter quantum field operators (anti)commute). Thus topology, generally conceived as the study of properties of Euclidean, undirected spatial connections between points of space, seems to fare poorly against the inherently causal or temporal and directed nature of the connections between events that relativity theory 
brought to light [47, 4, 15, 28]. To emphasise the fundamentally causal or temporal, as opposed to spatial or topological proper, interpretation of our models, we may summarise our approach to the following: we study the dynamics and coherent quantum interference of causality, hence of the topology of spacetime, rather than merely of the topology of space. For this, it is perhaps more accurate to call the topology to be considered here causal topology and more appropriate to conceive our scheme as an algebraic description of some kind of quantum causal foam.

Also from a quantum-theoretic point of view, the usual notion of topology seems rather inappropriate, for what does space(time) as an inactive pre-existent realm 'out there' - one that is independent of the dynamical relations between quanta and our 'measurement interactions' with them-mean in the quantum deep? To us the fundamental approach seems to be the other way around: it is the dynamical relations and interactions of quanta that define spacetime. In a more operationalistic note and in accord with the general philosophy of quantum mechanics that supports an observer dependent, or even created, physical reality, it is more likely that our own observations or measurements of the dynamics of quantum causality yields, in some conception and concomitant modelling of the process of measurement in the manner of Bohr's correspondence principle, the classical (and more familiar!) picture of spacetime as a topological manifold. Indeed, for discretised quantum spacetime topologies this limit construction and its interpretation as the emergence of classicality à la Bohr, has already been proposed [31]; here we only reuse it in the context of fluctuating dynamical quantum causal topology. At least it has been contended that the commutative algebras of continuous $\left(i e, C^{0}\right)$ coordinates of the events of spacetime as a topological manifold arise precisely at such a classical limit [29] with the latter being interpreted as a correspondence principle ̀̀ la [31.

Affine to the remarks above is the point that general relativity may be conceived as the dynamical theory of $g_{\mu \nu}(x)$, with $x$ living in a differential manifold $M$; hence, since the metric defines the local causal relations between events $\left(i e, g_{\mu \nu}(x)\right.$ delimits the lightcone at every event $x$ of $M$ ), gravity may be thought of as the dynamics of local causality - the dynamical tilting of the local lightcone due to the gravitational force. In turn, it is customary in physical jargon to call local causality 'locality', so that general relativity may be viewed as a sound dynamical theory of locality. The principle of locality in general relativity is successfully captured by the mathematical assumption that on top of $M^{\prime}$ 's topological manifold $\left(i e, C^{0}\right)$ structure, there is also a differential geometric or $C^{\infty}$-smooth structure which, in turn, renders $g_{\mu \nu}(x)$ a smooth field. In $M$ causality connects and dynamically evolves between infinitesimally separated events. That general relativity is a dynamical theory of the field of locality $g_{\mu \nu}$ is concisely encoded in the assumption of $M$ as a differential manifold. This $M$ seems to be the last classical vestige of an ether-like substance in our theories of spacetime structure and its dynamics [10 - a classical relic that seems to be in irreconcilable discord with the inherently granular and algebraic character of the quantum [11, 12]. Thus, it would be 
desirable that our algebraic and reticular models of quantum causal foam yield in the aforementioned limit not only the topological, but also the local differential structure of the classical spacetime manifold $M$ [31].

Related to these comments on locality is the relevant issue of spacetime event localisation. It has been amply justified that one cannot measure the local gravitational field $g_{\mu \nu}$ at an event $x$ with accuracy higher than Planck's space-time intervals $l_{P} \simeq 10^{-35} \mathrm{~m}$ $t_{P} \simeq 10^{-44} s$ without creating a black hole. This implies an innate granularity (ie, a built in cut-off) and an irreducible fuzziness in operations of spacetime localization at quantum scales plus it questions the validity of the assumption of a geometrical pointset manifold for spacetime. Indeed, it is well established that field theories like general relativity which have assumed a point-set spacetime continuum up-front are infested with singularities long before their quantisation becomes an issue. This unpleasant phenomenon becomes even more acute in the quantum field theories of matter in the absence of gravity which suffer from infinities that are predominantly due to the pointlike character of the sources of the fields involved, which sources, in turn, are thought of as occupying point events in the Minkowski continuum. All this seems to justify our primitive intuition of $a b$ initio substituting or 'smearing' the points of a manifold by larger or coarser open sets about them, which regions can also coherently superpose with each other - an intuition that lies at the heart of our approach to spacetime foam [38, 39, 31, 29, 24]. This resembles the operator valued distribution approach to the flat quantum field theories of matter, as well as the by now standard method of 'blowing up' singular points in algebraic geometry [30]. To wrap things up, we claim that locality and localisation are purely classical conceptions intimately related to the manifold model of spacetime that in a quantum theoresis of spacetime structure and gravity must be abandoned only to be recovered in some classical limit. It follows that our algebraic structures modelling quantum causal foam are not only reticular, but also in quite a strong sense alocal [31].

The present paper is organised as follows:

- In section 1 we present the algebraic model for spacetime foam mainly concentrating on a special example of a method, quite standard in algebraic geometry, of extracting (finitary) topological spaces from (finite dimensional) algebras.

- In section 2 we 'causalize' our algebras in the sense that we give a sound causal interpretation to the extracted finitary topologies and then give a sound quantum interpretation to these locally finite causal topologies so that the epithet spacetime foam given to them is justified.

- In section 3 we argue for a limit construction that recovers the classical spacetime continuum and the local differential structure of its events from an inverse system of these alocal dynamical quantum causal substrata. 
- In section 1 a toy model giving us different spacetimes as values of a quantum observable is presented.

The afterword concluding the paper attempts to anticipate and reply to some "natural' criticism that one may exercise on our algebraic approach to spacetime foam, as well as it gives a brief account of what in our view will more likely be the future development of the theory.

\section{$1 \quad$ Algebraic model}

Let us now move to technicalities. Following a very general operationalist philosophy, with any (in particular, spacetime) measurement we associate an 'operation device' as it is common practice in standard quantum mechanics; that is to say, a self-adjoint operator in an appropriate Hilbert space. The eigenvalues of the operator are the possible outcomes of acts of measurement on the quantum system in focus [, and its eigenspaces are the 'rooms' where the state of the system jumps after measurement. Note that, as a matter of fact, it is not relevant what particularly is drawn on the device's scale. This means that instead of a self-adjoint operator we can consider just a partition of the overall space into mutually orthogonal subspaces generated by an appropriate decomposition of the unit operator in the algebra of observables $\mathbf{1}=\sum_{\alpha \in A} P_{\alpha}$. So, if we would have a procedure which associates with every summand in the above partition a topological space rather than a number, then we could say that topology has become a quantum observable. To be able to do that, we have to assume that the state space itself must possess some additional structure.

According to our settings this additional structure is that of an algebra, associative but not commutative in general. In this section we describe the machinery for constructing topological spaces out of algebras which consists of:

- Spatialisation procedure. Given a (finite dimensional) algebra, it extracts the set of points and endows it with the Rota topology.

- Geometrisation. We show that geometrical properties, such as the differential structure or 'differentiability', are captured already at the finitary level, so that the $C^{\infty}$-smooth continuum is not a necessary prerequisite for their existence.

- Functoriality. We establish the duality between the algebras and appropriate geometries which enables us to build the limits which, in turn, are intended to support the classical correspondence principle. We address this issue in section 3 .

\footnotetext{
${ }^{1}$ In this work we take it almost axiomatically that spacetime is a quantum system [31.
} 


\subsection{Instead of manifolds}

We pass from the raison d'être for reticular spacetimes presented in the previous section to their façon d'être. As it was claimed above, for every outcome of a pragmatic spacetime measurement we expect the result to be formulated in finitary terms. The idea to consider gravitational spacetime models consisting of finite number of points arises in Regge [32], then at the level of topological spaces it was elaborated by Isham 19] who suggested a lattice of finite topologies to serve as the (kinematical) configuration space for a canonical (ie, Hamiltonian) topodynamics. To what extent can finite topological spaces be similar to manifolds? To give this question a precise meaning we recall the coarse-graining procedure or 'algorithm' due to Sorkin [38].

Finitary substitutes of topological spaces. From a formal point of view and briefly, Sorkin's algorithm looks as follows. When we are speaking of spacetime as a topological or $C^{0}$-manifold $M$, its mere definition assumes that we have a covering or charting $\mathcal{C}$ of $M$ by open subsets. The idea of coarse-graining is to replace the existing topology of the manifold $M$ by that generated by the covering $\mathcal{C}$. At the heart of this idea lies the primitive intuition that 'large', 'coarse' or 'fuzzy' open sets are more 'pragmatic' than points [38, 39, 31, 7, 29], so that the geometric point-like continuum $M$ will be recovered at the ideal limit of infinite refinement of the $\mathcal{C}$ s. As a result, the spacetime manifold acquires the cellular or simplicial structure with respect to $\mathcal{C}$, so that the events belonging to one cell are thought of as operationally indistinguishable. Then, instead of considering the set $M$ of all events we can focus on its quotient with respect to the relation

$$
x \equiv y \quad \text { if and only if } \quad \forall \mathcal{O} \in \mathcal{C} \quad x \in \mathcal{O} \Leftrightarrow y \in \mathcal{O}
$$

which is finite whenever the atlas $\mathcal{C}$ of $M$ is finite.

Let us consider the behaviour of sequences of elements in finite topological spaces. First it is worth mentioning that if a finite topological space is Hausdorff, then its topology is necessarily discrete ( $i e$, in a sense degenerate). Since we are going to deal with non-trivial topologies, we should not expect them to be Hausdorff or $T_{2}$. As a consequence, the theorem of the uniqueness of the limit of a sequence will not be valid anymore. Another equivalent way of defining a topology $\tau$ on a set $X$ is to define which sequences in $X$ converge with respect to $\tau$ and which do not. So, for finite $X$ we can instead of listing out or drawing pictures of open sets draw (or describe somehow) the graph of convergences of sequences $x, x, \ldots x, \ldots \rightarrow y$, this indicating that the constant sequence $\{x\}$ converges to $y$ in the topology $\tau$. Consider a couple of examples in which, to distinguish between open and closed intervals, we write

$$
\begin{aligned}
& (a, b)=\{x: a<x<b\} \\
& {[a, b]=\{x: a \leq x \leq b\}}
\end{aligned}
$$


Example 1. Let $M$ be a piece of plane: $M=(0,1) \times(0,1)$, and $\mathcal{C}=\left\{\mathcal{O}_{1}, \mathcal{O}_{2}, \mathcal{O}_{3}\right\}$ be its covering with $\mathcal{O}_{1}=\left(0, \frac{2}{3}\right) \times\left(\frac{1}{3}, 1\right), \mathcal{O}_{2}=\left(\frac{1}{3}, 1\right) \times\left(\frac{1}{3}, 1\right)$ and $\mathcal{O}_{3}=(0,1) \times\left(0, \frac{2}{3}\right)$ (See Fig. 目)

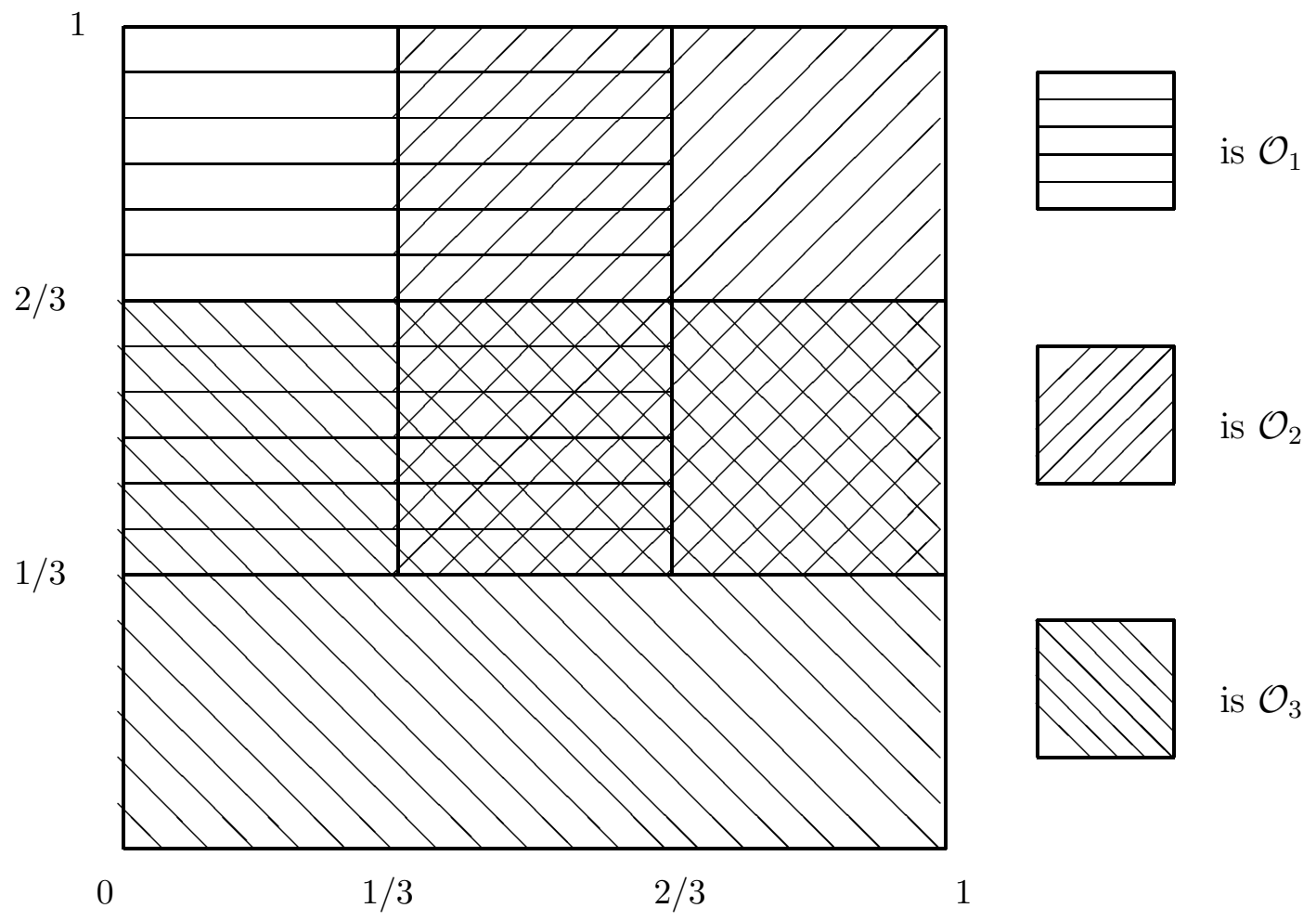

Figure 1: The covering of a piece of plane.

Let us describe in detail how the convergence graph (Fig. 2a) is built. Denote by subsequent numbers the following subsets of $M$

$$
\begin{array}{cl}
1=\left(0, \frac{1}{3}\right) \times\left(\frac{2}{3}, 1\right) & 4=\left(0, \frac{1}{3}\right) \times\left[\frac{1}{3}, \frac{2}{3}\right] \\
2=\left(\frac{1}{3}, 1\right) \times\left(\frac{2}{3}, 1\right) & 5=\left[\frac{1}{3}, \frac{2}{3}\right] \times\left(\frac{2}{3}, 1\right) \\
3=(0,1) \times\left(0, \frac{1}{3}\right) & 6=\left(\frac{2}{3}, 1\right) \times\left[\frac{1}{3}, \frac{2}{3}\right] \\
7=\left[\frac{1}{3}, \frac{2}{3}\right] \times\left[\frac{1}{3}, \frac{2}{3}\right]
\end{array}
$$

which stand for the equivalence classes of the relation (1).

The arrows drawn on Fig. 2a appear as follows. Take, say, a point in the set 1 and a point in the set 4 . Then any set from $\mathcal{C}$ containing 1 contains 4 , therefore 1 tends to 4, which is depicted by drawing the appropriate arrow. All other arrows are obtained likewise. 
Example 2. A circle. Let $M=\exp (i \phi)$, and let the covering be $\mathcal{C}=\left\{\mathcal{O}_{1}, \mathcal{O}_{2}, \mathcal{O}_{3}\right\}$ with

$$
\begin{aligned}
& \mathcal{O}_{1}=(-\pi / 3,5 \pi / 6) \\
& \mathcal{O}_{2}=(\pi / 2,9 \pi / 6) \\
& \mathcal{O}_{3}=(-2 \pi / 3, \pi / 3)
\end{aligned}
$$

for which the convergence graph is built in a similar way and shown on Fig. 2hb.

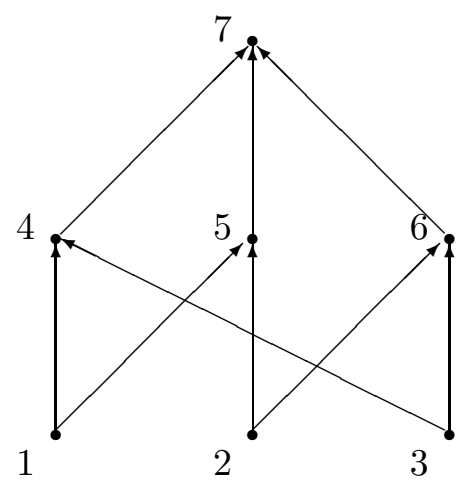

a). The plane.

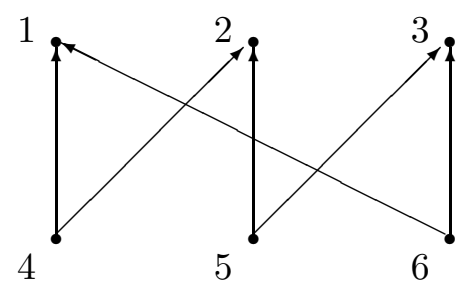

b). The circle.

Figure 2: The graphs of convergences for the finitary substitutes for the plane and the circle. The numbers label the vertices to concord with the matrix representations (6).

It is straightforward to prove that for any points $x, y, z$, of a finite topological space $X x \rightarrow y$ and $y \rightarrow z$ imply $x \rightarrow z$. That means that its graph of convergences, denoted by $X$, will be always transitive. This remarkable property of finitary substitutes makes it possible to associate algebras with them, called Rota algebras.

\subsection{Rota algebras}

Rota algebras in Dirac notation. Let $X$ be a finite topological space. Consider the linear space $\Omega$ whose basis $|i\rangle\langle j|$ is labelled by tending pairs $i \rightarrow j$ of points of $X$.

$$
\Omega(X)=\left\{\sum_{i, j \in X}|i\rangle\langle j| \text { such that } i \rightarrow j\right\}
$$

In the sequel, when no confusion occurs, we omit the notation of the topological space $X$ in parentheses and simply write 


$$
\Omega=\Omega(X)
$$

Define the product on $\Omega$ by setting it on its basic elements:

$$
|i\rangle\langle j \| k\rangle\langle l|= \begin{cases}|i\rangle\langle l| & , \text { if } j=k \\ 0, & \text { otherwise }\end{cases}
$$

Note that $|i\rangle\langle l|$ in (3) is always well-defined since the relation of convergence " $\rightarrow$ " is always transitive, that is why the existence of darts $i \rightarrow j$ and $j \rightarrow k$ in the convergence graph of $X$ always enables the existence of $i \rightarrow k$. The space $\Omega$ with the product (3) is called the Rota ALGEBRA of the topological space $(X, \rightarrow)$. These algebras were first introduced in 35] in the context of combinatorial theory.

The matrix representation of Rota algebras. Given the Rota algebra of a finite topological space $X$, its standard matrix representation is obtained by choosing the basis of $\Omega$ consisting of the elements of the form $|i\rangle\langle k|=\mathbf{e}_{i k}$, with $i k$ ranging over all converging pairs $i \rightarrow k$ of elements of $X$. The matrices $\mathbf{e}_{i k}$ (called matrix units) are defined as follows:

$$
\mathbf{e}_{i k}(m, n)= \begin{cases}1 & m=i \text { and } n=k \text { (provided } i \rightarrow k) \\ 0 & \text { otherwise }\end{cases}
$$

where $\mathbf{e}_{i k}(m, n)$ stands for the element in the $m$-th row and the $n$-th column of the matrix $\mathbf{e}_{i k}$. We can also extend the ranging to all pairs of elements of $X$ by putting $\mathbf{e}_{i k} \equiv 0$ for $i \not \rightarrow k$. Then the product (3) reads:

$$
\mathbf{e}_{i k} \mathbf{e}_{i^{\prime} k^{\prime}}=\delta_{k i^{\prime}} \mathbf{e}_{i k^{\prime}}
$$

To specify a Rota algebra in the standard matrix representation we fix the template matrix replacing the unit entries in the incidence matrix $I_{i k}$ of the graph $X$ :

$$
I_{i k}= \begin{cases}1 & i \rightarrow k \\ 0 & \text { otherwise }\end{cases}
$$

by wildcards $*$ ranging independently over all numbers. For the examples considered above the templates for their Rota algebras have the following form: 


$$
\Omega(\text { plane })=\left(\begin{array}{ccccccc}
* & 0 & 0 & * & * & 0 & * \\
0 & * & 0 & 0 & * & * & * \\
0 & 0 & * & * & 0 & * & * \\
0 & 0 & 0 & * & 0 & 0 & * \\
0 & 0 & 0 & 0 & * & 0 & * \\
0 & 0 & 0 & 0 & 0 & * & * \\
0 & 0 & 0 & 0 & 0 & 0 & *
\end{array}\right) \quad ; \quad \Omega(\text { circle })=\left(\begin{array}{cccccc}
* & 0 & 0 & 0 & 0 & 0 \\
0 & * & 0 & 0 & 0 & 0 \\
0 & 0 & * & 0 & 0 & 0 \\
* & 0 & * & * & 0 & 0 \\
* & * & 0 & 0 & * & 0 \\
0 & * & * & 0 & 0 & *
\end{array}\right)
$$

where the wildcard * denotes the ranging over the field of numbers; for instance, the algebra associated with the two-point space with the topology $\bullet \longrightarrow \bullet$ has the the following template matrix

$$
\Omega(\rightarrow)=\left(\begin{array}{ll}
* & * \\
0 & *
\end{array}\right)=\left\{\left(\begin{array}{ll}
a & b \\
0 & c
\end{array}\right) \mid a, b, c \in \mathbf{C}\right\}
$$

So, we see that any finite topological space can be described in terms of a finitedimensional algebra (for more details we refer to [45]).

\subsection{Spatialisation and Rota topology}

Here we show how to associate a finite topological space with an arbitrary finitedimensional algebra.

The emergence of points. Let us start with a given finite-dimensional associative (and non-commutative, in general) algebra $\Omega$. According to standard conceptions and methods of modern algebraic geometry, as well as the general algebraic approach to physics, we assume points to be irreducible representations (IRs) of $\Omega$. Let us dwell on this issue in a bit more detail. All the contents of our paper respects the so-called 'observability principle'. This means that the spatialisation (viz. the emergence of points) should be considered with respect to the measurements we possess. This, in turn, is supposed to be captured by the notion of an algebra $\Omega$ of observables available for experimental discourse with the quantum system in focus (here, spacetime). Starting from this algebra we have to introduce the notion of points as something to be regarded as 'undivisible', or 'inseparable', or even 'atomic' - an irreducible 'ur-element' from a set-theoretic or purely geometric point of view. From an algebraic perspective, as it is generally assumed, irreducible representations (IRs) of the algebra of observables involved seem to meet best this 'elementarity of points' requirement. So, the first step of the spatialisation procedure is creating (or finding) points:

$$
\{\text { points }\}=\{\text { IRs }\}
$$


Standard set with nonstandard topology. When the first spatialization step in a standard way (7) is done we may wish to proceed by endowing the set of points with a topology. There are standard recipes for this step as well like, say, the Zariski topology on the prime spectrum of $\Omega$. Unfortunately, on finite-dimensional algebras this topology is always discrete, which leaves us no chance to fit the above requirement of being non-Hausdorff ( $i e$, not $T_{2}$ ). So, we are compelled to find another topology.

As it was mentioned above, to define a topology on a finite set, it is enough to define it in terms of convergences, namely, to state which point tends to which [20]. These terms are the most appropriate for describing the Rota topology (first it was introduced in 450).

Let $\Omega$ be a finite-dimensional algebra. Denote by $X$ the set of points of $\Omega$, each of which we shall associate with a prime ideal in $\Omega$. Consider two points (representations of $\Omega) x, y \in X$ and denote by the same symbols $x, y$ their kernels as it produces no further confusion. Both of them, being kernels of representations, are two-sided ideals in $\Omega$, in particular, subsets of $\Omega$, hence both of the following expressions make sense:

$$
x \cap y \subset \Omega \quad \text { and } \quad x \cdot y \subset \Omega
$$

the latter denoting the product of subsets of $\Omega: x \cdot y=\{a \in \Omega \mid \exists u \in x, v \in y: u v=$ $a\}$. Since $x, y$ are ideals, we always have the inclusion $x \cdot y \subseteq x \cap y$ which may be strict or not. Define the relation $\propto$ on $X$ as follows:

$$
x \propto y \quad \text { if and only if } \quad x y \neq x \cap y
$$

Then the Rota Topology is the weakest one in which $x \propto y$ implies the convergence $x \rightarrow y$ of the point $x$ to the point $y$. Explicitly, the necessary and sufficient conditions for $x$ to converge to $y$ in the Rota topology reads:

$$
x \rightarrow y \quad \text { if and only if } \exists y_{0}, \ldots, y_{n} \mid y_{0}=x, y_{n}=y ; y_{k-1} \propto y_{k}
$$

This operation is called the transitive closure of the relation $\propto$. Note that, in general, the Rota topology can be defined on any set of ideals.

It was proved by Stanley 41] that in that particular case when $\Omega$ is the Rota algebra of a finite topological space, then its spatialisation $X$ endowed with the Rota topology (9) is homeomorphic to the initial topological space. However, in general if we have two finite topological spaces and a continuous mapping between them, their Rota algebras will not be homomorphic. Recently, 'good' classes of topological spaces were discovered for which the transition to Rota algebras is functorial [6, 46]. In our approach, it supports the existence of the classical limit. We return to this issue in section 3 . 


\subsection{Graded structures and geometrisation}

Here we discover that the topological spaces which arise in Sorkin's finitary substitution procedure are typically 'good' from the point of view of their Rota algebras. We show that the finite-dimensional analogues of differential calculi can be introduced on finite topological spaces as well.

Basic definitions. The 'good' topological spaces we mentioned above are abstract simplicial complexes. They are defined as follows. Let $V$ be a non-empty finite set. Call the elements of $V$ vertices. A collection $\mathcal{K}$ of non-empty subsets of $V$ is called (abstract) SIMPLICIAL COMPLEX with the set of vertices $V$ whenever

- $\forall v \in V \quad\{v\} \in \mathcal{K}$

- $\forall P \in \mathcal{K}, \forall Q \subseteq V \quad Q \subseteq P \Rightarrow Q \in \mathcal{K}$

The elements of $\mathcal{K}$ are called simplices. The topology on $\mathcal{K}$ is defined in terms of convergences:

$$
P \rightarrow Q \quad \stackrel{\text { Def }}{\Leftrightarrow} \quad P \subseteq Q
$$

where the latter inclusion makes sense since $P, Q$ are subsets of $V$.

Now let $M$ be a topological space and $\mathcal{C}$ an arbitrary covering of it (call the elements of $\mathcal{C}$ charts). With any such $\mathcal{C}$ a simplicial complex can be associated according to the following definion. A NERVE of the covering $\mathcal{C}$ is a simplicial complex $\mathcal{K}(\mathcal{C})^{2}$ such that

$$
\begin{aligned}
& V=\mathcal{C} \\
& P \in \mathcal{K} \Leftrightarrow \cap P \neq \emptyset
\end{aligned}
$$

Here the first condition (10) means that the vertices of $\mathcal{K}$ are associated with the charts of the covering $\mathcal{C}$, and the second condition (10) means that the simplices $P$ of $\mathcal{K}$ are the sets of charts of $\mathcal{C}$ whose intersection is non-empty.

So, given a covering of $M$, we can in principle form two finitary topological spaces out of it: the finitary substitute $X$ described in section 1.1 and the nerve $\mathcal{K}$ of $\mathcal{C}$. In the case when the covering

- $\mathcal{C}$ is minimal (ie, when we cannot delete any of its elements for $\mathcal{C}$ to remain a covering) and

\footnotetext{
${ }^{2}$ This so-called 'nerve-construction' of simplicial complexes from (open) covers of a topological space is originally due to Cech [9]; see also [1]. We wish to thank the referee of Classical and Quantum Gravity for bringing to our attention this fact.
} 
- generic (ie, when all nonempty intersections are different),

these two topological spaces are the same. To show it, note that the minimality enables the first condition (10). Then with any equivalence class (1) of points of $M$ we associate the following simplex $P \in \mathcal{K}$ :

$$
P=\{A \in \mathcal{C} \mid P \subseteq A\}
$$

and the second condition $(\sqrt{10})$ for $\mathcal{C}$ to be generic enables this correspondence to be one-to-one.

From now on we assume that finite topological spaces $X$ we are dealing with are simplicial complexes. Let us study the structure of their Rota algebras. First note that $X$ is a graded set, namely, with any simplex its DIMENSION is associated:

$$
\operatorname{dim} P=\operatorname{card} P-1
$$

- the number of its vertices minus one.

Denote by $\mathcal{K}^{n}$ the $n$-skeleton of $\mathcal{K}$ - the set of its simplices of dimension $n, \mathcal{K}^{n}=\{P \in$ $\mathcal{K}: \operatorname{dim} P=n\}$ and consider the linear spans

$$
\mathcal{H}^{n}=\operatorname{span} \mathcal{K}^{n}=\left\{\sum_{P \in \mathcal{K}^{n}} c_{P}|P\rangle\right\}
$$

The BORDER OPERATOR $\delta: \mathcal{H}^{n} \rightarrow \mathcal{H}^{n-1}$ acts as

$$
\delta|P\rangle=\sum_{v \in P} \epsilon_{v P}\left|P_{v}\right\rangle
$$

(where $\epsilon_{v P}= \pm 1$ are the appropriate incidence coefficients) and then extends to the space $\mathcal{H}$ (assuming $\delta|v\rangle=0, \forall v \in V)$ :

$$
\mathcal{H}=\oplus \mathcal{H}^{n}=\operatorname{span} \mathcal{K}
$$

Taking advantage of the Dirac notation, the same symbol $\delta$ can be used to denote its adjoint, called the COBORDER OPERATOR, which acts from $\mathcal{H}^{* n}$ to $\mathcal{H}^{* n+1}$, as follows

$$
\forall P \in \mathcal{K}^{n} \quad\left\{\begin{array}{llll}
\langle P| \delta & \in & \mathcal{H}^{* n+1} \\
\delta|P\rangle & \in & \mathcal{H}^{n-1}
\end{array}\right.
$$


The Rota algebra $\Omega(\mathcal{K})$ defined in (2) also has a graded structure, namely with each basic element $|P\rangle\langle Q|$ the number

$$
\operatorname{deg}|P\rangle\langle Q|=\operatorname{dim} Q-\operatorname{dim} P
$$

is associated. It follows directly from the multiplication rule (3i) that deg is really a grading. Thus, $\Omega(\mathcal{K})$ can be written as a direct sum of its graded subspaces in the following manner

$$
\Omega(\mathcal{K})=\bigoplus_{i} \Omega^{i} ; \quad \Omega^{i}:=\operatorname{span}_{\mathbf{C}}\{|P\rangle\langle Q|: \operatorname{deg}| P\rangle\langle Q|=i\}
$$

The differential structure on $\Omega(\mathcal{K})$. Let us show that $\Omega(\mathcal{K})$ always has the structure of a differential module over $\mathcal{A}$, namely, that induced by the projection $\sigma$ onto the quotient. Now let us explicitly provide the form of the differential d on $\Omega(\mathcal{K})$. The following theorem holds (for the proof we refer to [46]).

The differential in the incidence algebra $\Omega(\mathcal{K})$ of a simplicial complex $\mathcal{K}$ has the following form. Let $|P\rangle\langle Q| \in \Omega^{n}$, then

$$
\mathrm{d}|P\rangle\langle Q|=| \delta P\rangle\left\langle Q\left|-(-1)^{n}\right| P\right\rangle\langle Q \delta|
$$

where $\delta$ is the symbol (12)-(13) for both border and coborder operations.

So, we have established that 'good' coverings capture not only the $C^{0}$-topology (as it was shown by Sorkin [38], see also [29]), but they also possess the differential structure similar to the module of differential forms. Namely,

- The rôle of the algebra of smooth functions is played by the diagonal part (or, in other words, of the component of degree 0) - denote it $\mathcal{A}$ - of its Rota algebra.

$$
\mathcal{A} \equiv \Omega^{0}
$$

- The $k$-degree component $\Omega^{k}$ of the Rota algebra plays the rôle (ie, has the same modular properties with respect to the algebra $\mathcal{A}$ ) as the module of smooth differential forms of order $k$.

- The operator d (14) is the finite-dimensional analog of the Cartan-Kähler differential. 


\section{Classical and quantum causality}

As promised in the physical motivation opening the paper, in this section we intend to replace the finitary topologies described above by mathematically equivalent structures whose physical interpretation however is distinctly chrono-logical, or more generally, causal, rather than simply topo-logical or spatial. Then we will 'algebraicise' the resulting causal spaces à la Rota, thus arrive swiftly at structures that may be soundly interpreted as quantum causal spaces supporting quantum causal topologies. Thus we will possess a concrete theoretical (ie, structural-mathematical) paradigm of quantum causal foam.

Classical causal sets. First, we recall the 'classical' causal sets from [4 (the epithet 'classical' for the causal sets of Bombelli et al. will be justified shortly). An 'abstract' CAUSAL SET $\vec{F}$ is a locally finite partially ordered set (poset) whose partial order is physically interpreted as the causal 'after' relation between its event-vertices. Local finiteness pertains to the technical trait that every Alexandrov interval $A(x, y):=$ $\{z: x \rightarrow z \rightarrow y\}$ in $\vec{F}$ is finite. This must be compared with the 'finitarity' property of the posets modelling finite topological spaces obtained from locally finite open coverings $\mathcal{C}$ of a bounded region $X$ of a spacetime topological manifold $M$ by Sorkin's algorithm 38 as it was briefly discussed in 1.1. One easily verifies that the latter posets are a fortiori locally finite in the causal sets' sense. Thus, one may consider a region $X$ bounded both in space and in time in a (possibly curved) spacetime manifold $M$ motivated mainly by the 'pragmatic' fact that all our spacetime experiments are of finite spatial extension and temporal duration, as well as that in the course of them we record a finite number of events 31.

The causal reinterpretation of the posets in [38] from topological to causal rests on the assumption that the open covers of $X$ now represent coarse (because dynamically and quantally perturbing) approximations ('observations' or 'measurements') of the causal relations between the events of $X$ rather than between their spatial topological relations proper [28, 24]. Convincing arguments supporting such a reinterpretation of the relevant poset structures from topological to causal were originally given in [39. Subsequently in [28], this reinterpretation was coined causalisation. Causalisation was somehow forced on us by the theory of relativity which mandates that the physical connections between events are the causal ones, not the tachyonic, spatial or space-like ones 15.

Quantisation of causality. Of course, if our mathematical models of finitary causal spaces were merely 'arrow structures' (ie, essentially small poset categories or arrow semigroups, or even monoids) they would only allow for serial concatenations of causal arrows to form causal paths, but would not be able to model coherent superpositions of them. To actually model quantum interferences of causality we would have 
to pass from poset to algebra structures. Indeed, if we were able to somehow associate algebras with the locally finite posets modelling causal sets and interpret their formal '+' operation as coherent quantum superposition of the latter's causal arrows, we would arrive straightforwardly to structures which can be interpreted as quantum causal sets in much the same way that Sorkin's finitary topological spaces [38] were algebraicised and concomitantly quantised in [31]. We do it by associating Rota algebras with Bombelli's et al. causal sets like when we associated incidence algebras to finitary topological posets in section 1, as well as to study their finite dimensional irreducible representations (IRs) as befits quantum systems with finitely many degrees of freedom. The passage from a locally finite poset modelling a 'classical' causal set $\vec{F}$ to its associated incidence Rota algebra (2)

$$
\vec{\Omega}=\Omega(\vec{F})
$$

treated as QUANTUM CAUSAL SET $\vec{\Omega}$ introduced in $[28$ was coined 'quantisation of causality'. As it was also mentioned in connection with (3) in 1.2, the transitivity of the basic partial order relation defining a causal set enables us to define an associative product structure in its associated incidence algebra (ie, quantum causal set).

It must be stressed however that the aforementioned algebraisation of finitary causality achieved by passing from the non-interfering causal sets of Sorkin et al. to the quantally superposing elements of their associated incidence Rota algebras modelling quantum causal sets is by no means the sole 'justification' for studying the latter in the context of dynamically variable quantum causal topology and, in extenso, quantum gravity. Another issue of crucial importance is our ability to regard such structures as sound models of local causality - the potent quantal aspects of such algebraic models aside. That is, the principal (perhaps the sole!) dynamical variable in the quantum spacetime deep is local quantum causality.

In an inherently finitistic and algebraic setting like ours locality cannot afford a meaning similar to the one it connotes in the context of the classical differential geometric continuum model for spacetime adopted exclusively as a realm in which the differential equations for gravity may be meaningfully (in fact, uniquely!) written down. Instead of conceiving of gravitational actions as being represented by a $C^{\infty}$ smooth field of 'local causality' or 'locality', $g_{\mu \nu}(x)$, dynamically evolving between infinitesimally separated events in a differential manifold spacetime $M$, one may think of causal influences in a reticular 'environment' that propagate between immediately separated or contiguous events. In other words, in a finitary context the dynamical field of locality must somehow connect nearest neighbouring events, that is to say, events that define null Alexandrov intervals $A(x, y)=\emptyset$. Indeed, in [28], and based on an argument given in [5], it has been held that what is in fact subject to quantisation and, concomitantly, dynamical variability, is the generating or covering relation of the classical causal sets of Sorkin et al. Equivalently stated, only a reduction $\propto$ of the 
causally interpreted partial order $\rightarrow$ of the classical causal sets should be regarded as a quantum observable in the reticular-algebraic realm of quantum causal sets.

Quantum causality and sheaves. The significance of this assumption (the reduction of " $\rightarrow$ " to " $\propto$ ") should not be underestimated. For one thing, it lies at the heart of the sheaf-theoretic approach to curved quantum causality 24] as well as to the definition of a non-commutative topology for the latter [30]. This, without digressing much from our original theme, may be briefly described as follows: in section 3 we noted the (anti)functorial equivalence between Sorkin's finitary poset substitutes of continuous spacetime manifolds (or their equivalent simplicial complexes) and their associated incidence algebras [46]). In [29] it was first observed that the contravariant functor relating the poset category of finitary substitutes of Sorkin with the poset category of their associated incidence Rota algebras is, in fact, an example of a presheaf [7]. Subsequent sheafification of such presheaves gave rise to so-called finitary spacetime sheaves - algebraic spaces that may be interpreted as sound 'coarse' approximations of the algebras of continuous $\left(i e, C^{0}\right)$ spacetime observables.

The key structural feature of finitary spacetime sheaves is their (categorical) definition as local homeomorphisms between finitary topological posets and their associated incidence algebras, or in a causal context, between classical and quantum causal sets. Indeed, it has been extensively argued that the kinematics of quantum causal setswhich kinematics, in turn, stands for a locally finite, causal and quantal version of the kinematics of Lorentzian gravity - may be successfully modelled after non-trivial spin-Lorentzian connections defined on principal finitary spacetime sheaves of them and of their local gauge symmetry groups [24, 30]. In turn, these connections may be thought of as encoding complete information about the dynamical variability of the local quantum causality relations $\propto$ between the elements of the objects in the respective categories, as well as they support a purely categorical version of the principle of general covariance of general relativity since the connections employed are indeed finitary spacetime sheaf morphisms [24, 30]. In turn, this may be regarded as a significant preliminary step towards and entirely algebraic (in fact, categorical!) description of quantum gravity [12, 8].

Comparison with Wheeler's spacetime foam. We conclude this section by pointing out a fundamental difference between our approach to quantum causal foam and the 'usual' conception of spacetime foam as originally conceived by Wheeler [43]. Apart from the aforementioned important difference between the non-relativistic, undirected and 'spatial' connotations of the usual mathematical term 'topology' and the relativistic, directed and 'temporal' undertones of the physical notion of 'causality', topology is

\footnotetext{
${ }^{3}$ We tacitly assume that the kinematical structure suitable for classical Lorentzian gravity is that of a principal fiber bundle over a Lorentzian spacetime manifold, with structure group $S L(2, \mathbf{C})$, and an $s l(2, \mathbf{C})$-valued connection $\mathcal{D}$ on it.
} 
usually understood as the mathematical description of the global, 'large scale' or gross features of space proper. Characteristically, Wheeler's definition of foam was as $d y$ namical variations and quantum fluctuations of global topological features of spacetime (that is, spacetimes differing in holes, handles etc). That means, for instance, that amplitudes could be assigned to transitions between different topologies of 'spacetimeas-a-whole'.

In contrast, here we are more interested in dynamics and quantum interferences of microlocality or microlocal causality, since we are supposed to dissect spacetime into its finest causal elements at Planck scales - a realm where the classical notion of differential locality of the spacetime manifold is suspected to be grossly inadequate vis-a-vis quantum effects, let alone its global continuity (topological) properties. It is perhaps more appropriate altogether to describe our scheme as being

- neither local - since locality inappropriately evokes ideas from the classical differential manifold model for spacetime,

- nor non-local - since we have not claimed yet any significant relevance of our models to the by now well established ideas on quantum non-locality,

but ALOCAL [31]. After all, quantum causal sets still respect a novel quantum sort of locality that is by no means dependent on the pathological background geometric smooth spacetime continuum [28, 29, 24, 30]. Perhaps it is in this new conception of a 'quantum locality' that lies the strength and the value of the sheaf-theoretic approach to curved quantum causality, for one is actually able to stitch appropriately the 'local experimental data' or 'local spacetime measurements' (ie, local sections of the curved finitary spacetime sheaves of quantum causal sets) and build the entire spacetime (sheaf) structure - topological, differential, indefinite metric etc from such local information 22, 23]. Thus sheaf theory seems to provide us with powerful mathematical tools for implementing Bombelli et al.'s [4] deep insight that "causality as a partial order determines the topological, differential and metric structure of a Lorentzian spacetime manifold' - one could say, the raison d'être of the causal set approach to quantum gravity (see also [36]).

Of course, as it was mentioned earlier, there still is some sense of 'globalness' into our theoresis of the topology of spacetime as being a quantum observable, since we fundamentally assume that the results of measuring the spacetime topology are not numbers per se, but 'entire' topological spaces - elements of the 'topology spectrum' of spacetime foam as it was described in section 1 .

\section{Complementarity and the classical limit}

The principal aim of this section is to present a way in which the algebras employed to model quantum causal foam may be thought of as encoding in their very structure 
some elements which may then be interpreted as manifestations of a kind of quantum indeterminacy. We will also discuss a mechanism by which the classical spacetime manifold of macroscopic experience and its local differential structure can emerge from such alocal algebraic quantum spacetime foam substrata.

Since we take it as a fundamental issue that spacetime itself is a quantum system 31] whose main property, its (local) causal topology, is a quantum observable, we will argue how the two quintessential concepts of the usual quantum theory of matter, namely, Bohr's complementarity and the correspondence principle can be formulated in an entirely algebraic and combinatorial spacetime setting like ours.

\subsection{Algebraic evidence for the uncertainty principle}

Our elaborations with complementarity will depict a finitary-algebraic analogue of the indeterminacy relations between the so-called complementary or conjugate position/momentum observables of matter quanta which are usually thought of as propagating on a classical spacetime continuum. That is, since both the $q$ and $p$ observables of the usual (relativistic) matter quanta are most often assumed to have a continuous spectrum, the (locally) Euclidean manifold $\mathbf{R}^{4}$ model for spacetime appears to be almost inevitable [7].

The idea that a version of quantum complementarity is built into our finitary algebraic structures - an 'innate indeterminacy' of our algebraic models of spacetime foam so to speak, may be briefly presented as follows: first we may recall from [28] that with every classical causal set $\vec{F}$ one may associate its incidence Rota algebra (quantum causal set, see section 2) $\vec{\Omega}$ in a way completely analogous to how we associated it with a finitary topological space in the beginning of section 1.2. To do it, recall that any partial order on a finite set, in particular, the causal one, determines a finite topology, called the order topology. The convergence in this topology is just the order relation on the set $\vec{F}$.

$$
\vec{F}=\{x \rightarrow y\} \quad \rightarrow \quad \vec{\Omega}(\vec{F})=\operatorname{span}\{|x\rangle\langle y|: x \rightarrow y \in \vec{F}\}
$$

Now, if we consider the events of $\vec{F}$ as some kind of configuration space for quantum causality, the Rota algebra $\vec{\Omega}$ acts on the linear span of the events of $\vec{F}$. The operators of the form $|x\rangle\langle x|$ may then be referred to as quantum points-events - they are the 'causal self-incidences' in the quantum causal set $\vec{\Omega}$ and, evidently, they correspond to the reflexivity relations in the underlying causal set $\vec{F}$.

Then, the 'points' in the quantum causal set $\vec{\Omega}(\vec{F})$ on which we impose the Rota topology, now the latter having a quantum causal interpretation [28], à $l a$ [1.3 are the (primitive) ideals in $\vec{\Omega}(\vec{F})$ (ie, in our case, the kernels of equivalence classes of IRs of $\vec{\Omega}(\vec{F}))$ of the form: 


$$
I_{x}=\operatorname{span}\{|y\rangle\langle z|:| y\rangle\langle z|\neq| x\rangle\langle x|\}
$$

each which can be decomposed into a sum $I_{x}=I_{x}^{P}+I_{x}^{M}$ with

$$
\begin{aligned}
I_{x}^{P} & =\operatorname{span}\{|y\rangle\langle z|:| x\rangle\langle x \| y\rangle\langle z|=0\} \\
I_{x}^{M} & =\operatorname{span}\{|y\rangle\langle z|:| x\rangle\langle x \| y\rangle\langle z| \neq 0\}
\end{aligned}
$$

For any $x$ all the elements of $I_{x}^{P}$ lie in the commutative subalgebra $\vec{\Omega}^{0}:=\operatorname{span}\{|x\rangle\langle x|\}$ of $\vec{\Omega}$. We regard them as operations of determination of pure 'position' states of events of discretised quantum spacetime [31]. Effectively, $\vec{\Omega}^{0}$ is the reticular and quantal analogue of the commutative algebra of coordinates or 'position' states of the point-events of the classical spacetime manifold.

Furthemore, the operational quantum physical interpretation given to the elements of the the linear subspaces $\vec{\Omega}^{i}(i \geq 1)$ of $\vec{\Omega}$ as operations of determinations of pure ' $m o$ mentum' states of events of discretized quantum spacetime concords with the fact that any pair of operations from $I_{x}^{P}$ and $I_{x}^{M}$ either have zero product, or do not commute. As for the latter there are precise algebraic Heisenberg commutation, the so-called 'indeterminacy', relations that mathematically model them, also in the case of quantum causal sets there are abstract commutation relations that may be thought of as modelling the abstract complementarity described above. They are of the following kind:

$$
\left[I_{x}, I_{y}\right]:=I_{x} \cdot I_{y}-I_{y} \cdot I_{x} \neq 0
$$

from which we may read the following 'innate uncertainty':

What constitutes a 'quantum point-event' $I_{x}$ in a quantum causal set consists of all quantum spacetime momentum and path actions $|y\rangle\langle z|$ that exclude or 'non-determine' the very position determination $|x\rangle\langle x|$ of the event-vertex $x$ in the underlying causal set $\vec{F}$.

This is an abstract expression of the usual $q-p$ complementarity relations for matter quanta propagating in a continuous spacetime, and on them the whole edifice of a noncommutative topology for curved quantum causality rests, since indeed the product ideal $I_{x} \cdot I_{y}$ enters in a fundamental way into the very definition of the generating relation $\propto$ of the Rota (quantum causal) topology (described in 1.3), now having a directly causal meaning as immediate quantum causality [28, 24, 30]. Furthermore, and here lies the mathematical versatility of our finitary algebraic models, it is our recognition of the $I_{x} \mathrm{~s}$ as standing for abstract quantum point-events that makes it altogether possible to define a non-commutative Rota causal topology in a mathematically rigorous and semantically sound way [25, 30, 26]. 


\subsection{The emergence of classicality: correspondence principle}

Our labours about the correspondence principle will essentially manifest how the classical spacetime of 'macroscopic experience ef, with the commutative coordinate algebras of its events and the cotangent spaces of differential forms soldered on the latter, may be thought of as arising at the ideal limit of infinite localisation of its point-events (or at the experimentally implausible or 'non-pragmatic' infinite power of resolution of spacetime into its point-events) [38, 31, 29].

Functoriality. As we have already mentioned in the end of section 1.3, an arbitrary continuous mapping $\pi: \mathcal{K}^{\prime} \rightarrow \mathcal{K}$ between two finite topological spaces $\mathcal{K}^{\prime}$ and $\mathcal{K}$ in general produces no homomorphism of their incidence algebras. However, if we restrict ourselves to spaces having the structure of simplicial complex, the situation becomes completely different. Namely, the correspondence $\mathcal{K} \mapsto \Omega(\mathcal{K})$ is a contravariant functor from the category of simplicial complexes to the category of differential modules over finite-dimensional commutative algebras. This dual mapping was first introduced in [2], and the functorial properties were studied in detail in [46]. It differs from the Eilenberg-Steenrod duality [9]-cf. (14).

In [38] inverse systems of finitary substitutes were considered which made it possible to reconstruct (or to have as a limit) a $C^{0}$-topological manifold space. Similarly, and from the categorically dual point of view of (algebras of) continuous $\left(i e, C^{0}\right)$ functions on the topological base spacetime manifold - the so-called 'algebras of continuous spacetime observables', in [29] inverse systems of finitary spacetime sheaves of continuous functions were also seen to 'converge' to the $C^{0}$-sheaf of continuous observables as the 'microscopic' power of resolution of spacetime into its point-events increased without bound. At this purely topological level there was no way one could introduce differential structures in intermediate finitary steps. Now, having this functoriality, we can consider direct limits of the Rota algebras of topological spaces. They are graded algebras, and the morphisms preserve grading as well as they intertwine with the Cartan-Kähler differential. This gives us the opportunity to treat intermediate finite spaces as full fledged spacetime substitutes, capturing both the topology (ie, 'convergeability') and the differential geometric structure (ie, 'differentiability').

When spacetimes are subsituted by finitary topological spaces, we may consider finer or coarser experiments. That is why we have to formalize the notion of refined experiment. Within the Sorkin discretisation procedure described in 1.1 a refinement means passing to an inscribed covering of the manifold. In this case any element of the finer covering is contained in an element of the coarser one.

Construction of classical limit. Each step of a limiting procedure, that is, of a refined covering, gives rise to a projection of appropriate complexes: the finer one is

\footnotetext{
${ }^{4}$ The inverted commas indicating that actually we have no experience of a spacetime continuum.
} 
projected to the coarser one. In the dual framework we have an injection of the smaller algebra associated with a coarser measurement to a bigger one associated with a finer one.

In general, limiting procedures for approximating systems (whatever these may be, posets or algebras) are organised using the notion of converging nets. Namely, each pragmatic observation is labelled by an index $\alpha$ and we have the relation of refinement $\succ$ on observations: $\alpha \succ \alpha^{\prime}$ means that the observation $\alpha$ is a refinement of $\alpha^{\prime}$.

When we are dealing with posets with each pair $\alpha, \alpha^{\prime}$ such that $\alpha \succ \alpha^{\prime}$ a canonical projection $\omega_{\alpha^{\prime}}^{\alpha}: \mathcal{K}_{\alpha} \rightarrow \mathcal{K}_{\alpha^{\prime}}$ is defined such that for any $\alpha \succ \alpha^{\prime} \succ \alpha^{\prime \prime}$ the following coherence condition holds:

$$
\omega_{\alpha^{\prime \prime}}^{\alpha}=\omega_{\alpha^{\prime \prime}}^{\alpha^{\prime}} \omega_{\alpha^{\prime}}^{\alpha}
$$

The functoriality requirement is evoked when

$$
\omega\left(\mathcal{K}_{\alpha}^{0}\right) \subseteq \mathcal{K}_{\alpha^{\prime}}^{0}
$$

Then we introduce the set of threads. A THREAD is a collection $\left\{t_{\alpha}\right\}$ of elements $t_{\alpha} \in \mathcal{K}_{\alpha}$ such that

$$
t_{\alpha}=\omega_{\alpha}^{\alpha^{\prime}} t_{\alpha^{\prime}}
$$

whenever $\alpha \succ \alpha^{\prime}$. Denote by $\mathbf{T}$ the set of all threads.

The next step is to make $\mathbf{T}$ a topological space which is done in a standard way [1]: $\mathbf{T}$ is a subspace of the total cartesian product $\mathbf{T}_{0}=\times_{\alpha} \mathcal{K}_{\alpha}$. Endow $\mathbf{T}_{0}$ with the product Tikhonov topology, then $\mathbf{T}$ being a subset of $\mathbf{T}_{0}$ becomes a topological space. Finally, we obtain the limit space $X$ as the collection of all closed threads from $\mathbf{T}$. This procedure is described in detail in [38].

A detailed scheme for building the dual limit - that of algebras was presented in [21]. As mentioned above, with any pair $\alpha \succ \alpha^{\prime}$ of pragmatic observations we have a canonical injection

$$
\omega_{\alpha}^{* \alpha^{\prime}}: \Omega\left(\mathcal{K}_{\alpha^{\prime}}\right) \rightarrow \Omega\left(\mathcal{K}_{\alpha}\right)
$$

Moreover, due to the requirement (18) the restriction of each $\omega^{*}$ on commutative subalgebras $\mathcal{A}=\Omega^{0} \subseteq \Omega$ is well defined. Now we first consider the set of all sequences

$$
\Omega=\times_{\alpha} \Omega\left(\mathcal{K}_{\alpha}\right)=\left\{\left\{a_{\alpha}\right\} \mid a_{\alpha} \in \Omega\left(\mathcal{K}_{\alpha}\right)\right\}
$$


and select the set of converging sequences in the following way. Note that $\mathcal{A}$ is an algebra. Introduce a norm $\|\cdot\|_{\alpha}$ in each finite-dimensional algebra $\Omega\left(\mathcal{K}_{\alpha}\right)$, then a sequence $\left\{a_{\alpha}\right\}$ converges if and only if for any $\epsilon>0$ there exists a filter $\mathcal{F}_{\epsilon}$ of indices $\alpha$ such that

$$
\forall \alpha, \alpha^{\prime} \in \mathcal{F}_{\epsilon} \quad \alpha \succ \alpha^{\prime} \Rightarrow\left\|\omega_{\alpha^{\prime}}^{* \alpha} a_{\alpha}-a_{\alpha^{\prime}}\right\|_{\alpha^{\prime}}<\epsilon
$$

Since any element of the limit algebra is a net, we can consider the coupling between the limit algebra and the limit space which consists of nets. The result of this coupling is a converging net of numbers whose limit may be thought of as the value of an element of the limit algebra at a point of the limit space. At present the rigorous results are obtained and verified only for the functorial part of the construction, for details the readers are referred to 46 .

Classicality in the limit. The conclusion of the last paragraph prompts us to first discuss briefly the mathematical character of the 'classical limit' or 'end space' alluded to above, before delving into the physical interpretation proper of the aforedescribed inverse or projective limit process on the inverse system or net of Rota algebras as well as into the physical nature of the end space that this procedure yields.

Perhaps the most important characteristic of the inverse limit space is that it can qualify as a manifold proper. We have already mentioned in section 1 in connection with Sorkin's poset discretisations of topological manifolds in [38] and in the beginning of this subsection that Rota algebras are objects (categorically) dual to those finitary posets. In the particular case that the latter are also (assumed to be) locally finite simplicial complex 'nerve-skeletonisations' of $C^{0}$-manifolds à la Čech-Aleksandrov [9, 1] as it is particularly of interest to us heref, the categorical duality between finitary posets/simplicial complexes and Rota algebras secures that, as an inverse system of finer-and-finer finitary poset topologies 'converges' to a space (ie, possesses an inverse limit space) that for all practical purposes is homeomorphic to ( $i e$, topologically indistinguishable from) a topological manifold [38, 29], so a projective system of their dual Rota algebras would also yield a $C^{0}$-manifold-like structure ${ }^{0}$.

Moreover, the bonus in our case is that the Rota algebras involved, being discrete differential spaces, encode information not only about the topology of the continuous space that they are supposed to substitute (and recover at a suitable projective limit), but also about its differential (or differentiable) attributes. In case we substitute a smooth manifold, one would then have strong reasons to believe, as we do, that the

\footnotetext{
${ }^{5}$ These are the 'good' posets alluded to in subsection 1.4.

${ }^{6}$ After all, it would be begging the question and it would put the terms 'approximations', 'substitutes' or 'replacements' to doubt, if one started with a topological manifold structure that one would like to approximate by finitary means - be it finitary posets as in 38] or their dual Rota algebras as here - and finally (ie, after the inverse limit procedure) one would recover a non-manifold topology!
} 
aforementioned end space would a fortiori be a $C^{\infty}$-smooth manifold, not just a $C^{0}$ continuous one. On the other hand, it is admittedly conceivable that although every 'approximating term' (Rota algebra) in the net may be 'niceๆ, the end space may turn out to be quite pathological ( $e g$, 'fractal'), or even more surprisingly, that while the maps between the approximating spaces are relatively regular, the limit space comes out rather singular. Certainly, we should not fully commit ourselves to recovering a smooth continuum; after all, we should allow for 'pathologies at the limit' for they would perhaps point at (potentially useful) features to be rectified at the finite level of the approximating spaces and their corresponding Rota algebras. Thus, perhaps it would be more appropriate instead of calling the Rota algebras involved 'discrete differential manifolds' - the reticular or combinatorial analogues of (the cotangent spaces of differential forms on) the $C^{\infty}$-smooth manifold [31, 24, 30], to coin them 'discrete differential spaces' - finitistic models of a differential geometry not necessarily identical to the usual calculus on smooth manifolds ( $i e$, the standard differential geometry)

More on the interpretational side, the suggested limit constructions play the crucial rôle of providing a firm (formal) algebraic background on which to (semantically) support the ever so important for the physical theory correspondence principle otherwise known as the method of making contact between the quantum and the classical models of the system in focus (here, spacetime). Thus, in closing this section, let us take the opportunity to recall from [31] and discuss briefly the physical interpretation given to these formal limit procedures as 'classical limit processes' - processes of emergence of the classical spacetime manifold of macroscopic scales from a wildly fluctuating primordial 'pool' (or 'soup'!) of alocal quantum causal topological foam substrata (always keeping in mind however the possibility mentioned in the previous paragraph that the limit space may not be free from 'differential abnormalities').

How classical is the classical limit? In [31] what principally motivated us to interpret the limit processes on discretized quantum spacetime topologies as Bohr's correspondence principle was the cogent quantum interpretation that the underlying Rota algebraic structures afforded, their prominent discrete differential geometric characteristics, as well as typical traits of the classical $C^{\infty}$-smooth spacetime manifold that were already foreshadowed at the reticular level of our structures. Thus we envisaged a process of 'decoherence' (or anyhow, of breaking or destruction of quantum coherence) of the topological arrow-connections in the alocal quantal (and here, causal) Rota algebras as our finitary experimenters employed higher power (ie, presumably higher microscopic energy) to resolve spacetime into its point-events, as it were, to localise it

\footnotetext{
${ }^{7}$ As we said in the previous footnote, for us, 'niceness' means 'the Rota algebra of a simplicial complex'; see section 1 .

${ }^{8}$ We would like to thank cordially the referee of Classical and Quantum Gravity for sensitising us to the possibility that apparently 'healthy' finitary approximations may yield at the limit pathological differential continua supporting rather singular differential calculi.
} 
maximally.

Being faithful to an operationalistic philosophy for physics and respecting the main lesson we have learned from applying quantum theory to spacetime structure (ie, that one cannot actually localize the gravitational field below Planck length without creating a singularity in the underlying spacetime manifold structure), we gathered that such maximal spacetime localisations are ideal (mathematical) constructions of non-

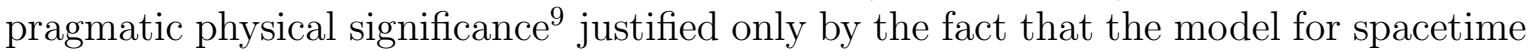
at large scales that works best (namely, the differential continuum) can be obtained at such a limit of infinite refinement of the (causal) foam pool. Indeed, since the local structure of classical spacetime (ie, the commutative coordinate algebras of its pointevents and the spaces of differential forms cotangent to the latter) is recovered at such an infinite localisation limit of the granular and quantal foam algebras, our identification of it with the usual correspondence principle (or limit) due to Bohr is well justified even if a posteriori it seems that our original motivation was quite 'ophelimistic' (ie, that the end-manifold somehow justifies the means-algebras exactly because of the existence of the mathematical limit constructions linking the two).

Altogether however, a 'die-hard' physicist may object to our 'emergence of classicality' scheme above by claiming that the classical spacetime manifold is the result of an actual physical process such as condensation for instance [15, 16, 3], so that a 'purely kinematical' (or structural) account of 'how come the continuum?' should give way to a more dynamical scenario. At the same time, a 'quantum purist' may also object to the way we use Bohr's correspondence principle in that, in quite a general sense, Bohr intended to use this notion in close connection with the process of measurement and the resulting recording of c-numbers from q-systems; while, strictly speaking, we argue here that, in fact, we measure or 'extract' entire topological spaces from quantum spacetime (see sections 1 and 2), not merely commutative numbers. Granted that we recognise that our algebraic description of spacetime foam has been characterised as being more 'kinematical' than 'dynamical'"ण and that we declared up-front that we regard the spacetime's (causal) topology as being a quantum observable, such criticism, although reasonably justified, could hardly present any significant hindrance to the further development of the theory of quantum spacetime structure propounded herein.

\section{A toy model}

In this section we present an explicit example of 49-dimensional Hilbert space endowed with a product operation, and a self-adjoint operator in this space such that for two its different eigenspaces the spatialisation procedure yields two topologically different

\footnotetext{
${ }^{9}$ Ever ready to reconsider however if and when experimentalists show us that one can actually record a real number in the laboratory.

${ }^{10}$ See Afterword in this paper, as well as [24, 30].
} 
spaces: a piece of plane and a circle considered in the example above.

Let $\mathcal{H}$ be the space of all complex valued $7 \times 7$ matrices:

$$
\mathcal{H}=M_{7}=\operatorname{span}\left\{\mathbf{e}_{i k} \mid i, k=1, \ldots, 7\right\}
$$

choosing matrix units introduced above in (4) as its orthonormal basis:

$$
\left(\mathbf{e}_{i k}, \mathbf{e}_{i^{\prime} k^{\prime}}\right)=\delta_{i i^{\prime}} \delta_{k k^{\prime}}
$$

The product operator on $\mathcal{H}$ which is necessary to define the Rota topology will be the usual matrix product: $\mathbf{e}_{i k} \cdot \mathbf{e}_{i^{\prime} k^{\prime}}=\delta_{k i^{\prime}} \mathbf{e}_{i k^{\prime}}$. Denote by $P_{i k}: \mathcal{H} \rightarrow \mathcal{H}$ the projector on the basis element $\mathbf{e}_{i k}$ and, denoting by $\operatorname{Proj}(a)$ the orthogonal projector on a unit vector $a$, define the following projectors:

$$
\begin{aligned}
& P_{c 2}=\operatorname{Proj}\left(\frac{1}{\sqrt{2}}\left(\mathbf{e}_{62}+\mathbf{e}_{72}\right)\right) \\
& P_{c 3}=\operatorname{Proj}\left(\frac{1}{\sqrt{2}}\left(\mathbf{e}_{63}+\mathbf{e}_{73}\right)\right) \\
& P_{p c}=\operatorname{Proj}\left(\frac{1}{\sqrt{179}}\left(\mathbf{e}_{11}-2 \mathbf{e}_{22}+3 \mathbf{e}_{33}-4 \mathbf{e}_{44}+6 \mathbf{e}_{55}+7 \mathbf{e}_{66}-8 \mathbf{e}_{77}\right)\right) \\
& P_{c c}=\operatorname{Proj}\left(\frac{1}{\sqrt{95}}\left(6 \mathbf{e}_{11}+5 \mathbf{e}_{22}+4 \mathbf{e}_{33}+3 \mathbf{e}_{44}+\mathbf{e}_{55}+2 \mathbf{e}_{66}+2 \mathbf{e}_{77}\right)\right)
\end{aligned}
$$

Consider the self adjoint operator $\mathcal{T}: \mathcal{H} \rightarrow \mathcal{H}:$

$$
\begin{aligned}
\mathcal{T} & =\left(P_{14}+P_{15}+P_{25}+P_{26}+P_{36}+P_{34}+P_{47}+P_{57}+P_{67}+P_{p c}\right)- \\
& -\left(P_{41}+P_{43}+P_{51}+P_{52}+P_{c 2}+P_{c 3}+P_{c c}\right)
\end{aligned}
$$

having three eigenvalues: $0,+1$ and -1 .

If we carry out the measurement associated with $\mathcal{T}$, the result will be 0,1 or 1. If the result is +1 , then we are in the subspace of $\mathcal{H}$ generated by the first line of (19). The algebra spanned on this subspace is isomorphic to the matrix algebra $\Omega$ plane from (6). When the eigenvalue is -1 , we get the eigenspace whose algebraic closure is isomorphic to $\Omega_{\text {circle }}$ from (6). When the eigenvalue is 0 , the span of the appropriate eigenspace is the full matrix algebra, and the resulting space consists of one point.

To clarify the constructions of this section let us consider in more detail what happens if we obtain the eigenspace associated with +1 eigenvalue. Begin with its algebraic closure. All the functions of the vector $\mathbf{e}_{11}-2 \mathbf{e}_{22}+3 \mathbf{e}_{33}-4 \mathbf{e}_{44}+6 \mathbf{e}_{55}+7 \mathbf{e}_{66}-8 \mathbf{e}_{77}$ will form the commutative algebra $\operatorname{Diag}_{7}$ of all diagonal $7 \times 7$ matrices:

$$
\operatorname{Diag}_{7}=\operatorname{span}_{\mathbf{C}}\left\{\mathbf{e}_{11}, \mathbf{e}_{22}, \mathbf{e}_{33}, \mathbf{e}_{44}, \mathbf{e}_{55}, \mathbf{e}_{66}, \mathbf{e}_{77}\right\}
$$


Then recall that according to (州) we have

$$
\begin{aligned}
\mathbf{e}_{14} \mathbf{e}_{47} & =\mathbf{e}_{17} \\
\mathbf{e}_{25} \mathbf{e}_{57} & =\mathbf{e}_{27} \\
\mathbf{e}_{36} \mathbf{e}_{67} & =\mathbf{e}_{37}
\end{aligned}
$$

and so we get the algebra $\Omega$ plane from (6). It is graded:

$$
\begin{aligned}
\Omega^{0} & =\operatorname{span}_{\mathbf{C}}\left\{\mathbf{e}_{11}, \mathbf{e}_{22}, \mathbf{e}_{33}, \mathbf{e}_{44}, \mathbf{e}_{55}, \mathbf{e}_{66}, \mathbf{e}_{77}\right\} \\
\Omega^{1} & =\operatorname{span}_{\mathbf{C}}\left\{\mathbf{e}_{14}, \mathbf{e}_{15}, \mathbf{e}_{25}, \mathbf{e}_{26}, \mathbf{e}_{36}, \mathbf{e}_{34}, \mathbf{e}_{47}, \mathbf{e}_{57}, \mathbf{e}_{67}\right\} \\
\Omega^{2} & =\operatorname{span}_{\mathbf{C}}\left\{\mathbf{e}_{17}, \mathbf{e}_{27}, \mathbf{e}_{37}\right\}
\end{aligned}
$$

The total algebra $\Omega$ is

$$
\Omega=\Omega^{0} \oplus \Omega^{1} \oplus \Omega^{2}
$$

It can be described by the template (6) (the right matrix). Let us spatialise it according to the recipe of section 1.3. There are 7 IRs of $\Omega$. To specify them, denote by $a=$ $\sum_{i k} a_{i k} \mathbf{e}_{i k}$ where the pairs $i k$ range over the values occurring in (20). Then the IRs of $\Omega$ are:

$$
\begin{array}{ll}
\mathbf{1}: a \mapsto a_{11} & \mathbf{5}: a \mapsto a_{55} \\
\mathbf{2}: a \mapsto a_{22} & \mathbf{6}: a \mapsto a_{66} \\
\mathbf{3}: a \mapsto a_{33} & \mathbf{7}: a \mapsto a_{77} \\
\mathbf{4}: a \mapsto a_{44} &
\end{array}
$$

Now let us describe the Rota topology on the set of the points (=IRs) that we obtained. First calculate the relation $\propto(8)$. Take two representations, say, 1 and 4 , and consider their kernels $x_{1}$ and $x_{4}$, and then their intersection $x_{1} \cap x_{4}$ and the product $x_{1} \cdot x_{4}$ :

$$
\begin{aligned}
& x_{1}=\left\{\sum_{i k} a_{i k} \mathbf{e}_{i k} \quad \text { such that } i k \neq 11\right\} \\
& x_{4}=\left\{\sum_{i k} a_{i k} \mathbf{e}_{i k} \quad \text { such that } i k \neq 44\right\} \\
& x_{1} \cap x_{4}=\left\{\sum_{i k} a_{i k} \mathbf{e}_{i k} \text { such that } i k \neq 11,44\right\} \\
& x_{1} \cdot x_{4}=\left\{\sum_{i k} a_{i k} \mathbf{e}_{i k} \text { such that } i k \neq 11,44,14\right\}
\end{aligned}
$$

and we see that $x_{1} \cap x_{4} \neq x_{1} \cdot x_{4}$, therefore according to (8) $x_{1} \propto x_{4}$. Checking in the same way that $x y \neq x \cap y$ (\&) for all pairs $i, k$ we get:

$\begin{array}{lll}1 \propto 4 & 1 \propto 5 & 2 \propto 5 \\ 2 \propto 6 & 3 \propto 4 & 3 \propto 6 \\ 4 \propto 7 & 5 \propto 7 & 6 \propto 7\end{array}$


Forming the transitive closure (9) of the relation $\propto$, we finally obtain the convergence graph drawn at figure 2 a). So, the topological space is reconstructed, let us study its differential properties.

The basic algebra $\mathcal{A}=\Omega^{0}$ is commutative, and the space of 'covectors' $\Omega^{1}$ possesses the structure of a (say, left) $\mathcal{A}$-module with the action of $\mathcal{A}$ defined by the multiplication (仿). This 'cotangent space' is 2-dimensional, namely, any element of $\Omega^{1}$ can be generated acting by the algebra $\mathcal{A}$ on the following two elements:

$$
\begin{aligned}
& \omega_{1}=\mathbf{e}_{14}+\mathbf{e}_{25}+\mathbf{e}_{36}+\mathbf{e}_{47}+\mathbf{e}_{57}+\mathbf{e}_{67} \\
& \omega_{2}=\mathbf{e}_{15}+\mathbf{e}_{26}+\mathbf{e}_{34}
\end{aligned}
$$

that is, $\Omega^{1}=\operatorname{span}_{\mathcal{A}}\left\{\omega_{1}, \omega_{2}\right\}$.

Similarly, the space of 2-forms is one-dimensional, its only generator is the element $\omega=\mathbf{e}_{17}+\mathbf{e}_{27}+\mathbf{e}_{37}$, so $\Omega^{2}=\operatorname{span}_{\mathcal{A}}\{\omega\}$. So, we see that the differential geometry of the substitute is really similar to that of a piece of a plane.

A similar construction can be carried out for the eigenspace of $\mathcal{T}$ associated with the value -1 . We remark that in this case the algebra $\mathcal{A}$ generated by the functions of the element $6 \mathbf{e}_{11}+5 \mathbf{e}_{22}+4 \mathbf{e}_{33}+3 \mathbf{e}_{44}+\mathbf{e}_{55}+2 \mathbf{e}_{66}+2 \mathbf{e}_{77}$ will have dimension 6 rather than 7 , namely:

$$
\mathcal{A}=\operatorname{span}_{\mathbf{C}}\left\{\mathbf{e}_{11}, \mathbf{e}_{22}, \mathbf{e}_{33}, \mathbf{e}_{44}, \mathbf{e}_{55}, \mathbf{e}_{66}+\mathbf{e}_{77}\right\} \simeq \operatorname{Diag}_{6}
$$

then, if we proceed in a similar way, we obtain the finitary substitute of a circle whose topology is depicted in figure 2 and whose Rota algebra has the template matrix (6).

\section{Afterword}

We conclude the present paper by commenting on six issues that one may legitimately bring up as critiques of the approach to spacetime foam presented above.

First, if the theoretical scheme ultimately aspires to be dynamical and at the same time alocal, one may reasonably ask: dynamics of quantum causal topology with respect to what? However, if there is such an external parameter space, or more colloquially, a background clock relative to which to refer the dynamical changes in quantum causality, is not this another manifestation of an ether-like absolute substance - another unobservable of the theory that is of dubious physical relevance? Our response to this is that each experimenter or observer of quantum causality creates her own picture of the dynamics of quantum causality; as it may, she creates her own 'quantum wristwatch' or 'quantum causal or time gauge', so that it is understood that a dynamics of 
quantum causality must be manifestly invariant or 'causal gauge covariant' relative to these gauges or 'local measurements' [24, 30.

Second, we must admit that as yet we have not been able to arrive at a full fledged dynamical scheme for spacetime foam and, in extenso, for quantum causality, so that it is perhaps more accurate to call our approach the kinematics of quantum causal topology [28, 24, 30]. For the closely related causal sets and their ultimate aim to model quantum gravity [4, 36, 37, 33], Sorkin has convincingly argued for the importance of first acquiring a firm grasp of the kinematics of a theory before tackling the problem of dynamics [39]. Overall, and having in mind the classical paradigm of the development first of the special and then of the general theory of relativity in that the former defined the kinematical structure of causality (ie, the Minkowski lightcone soldered at each event) while the latter formulated its localisation or gauging and concomitant curving ( $i e$, the dynamics of locality as encoded in $g_{\mu \nu}(x)$ ), it seems more reasonable to us to formulate first how quantum spacetime topology can possibly move (ie, its kinematical structure), and then entertain ideas of how it actually moves (ie, its dynamics). In this light, the correspondence limit manifold structure for spacetime that we are going to give should be properly called a kinematical mechanism for recovering the classical spacetime continuum.

Third, the use of non-*-algebras is often claimed to be unphysical. Related to the discussion above about kinematics versus dynamics is Penrose's [27] suggestion that "the true quantum gravity is a time asymmetrical theory". We also expect our theory to address somehow the problem of the quantum arrow of time vis-a-vis dynamical quantum causality and the related conception of spacetime foam. To begin with, the algebras that we use are non-involutive (ie, they are not $*$-algebras) unlike the algebras of observables of relativistic matter quanta used in quantum field theory over the flat Minkowski space. However, these field theories, as well as the classical field theory of gravity ( $i e$, general relativity), are manifestly time reversal invariant so that physics down to Planck length and duration $\left(l_{P}-t_{P}\right)$ appears to be predominantly time symmetric with the outstanding exception of the chiral, parity violating weak interactions at energies orders of magnitude lower than $E_{P}$. From an operational point of view the non-commutative *-algebras of quantum matter field observables employ the unary involution operation to model reversal of time, or more generally, of the sequential order of operation, and they are closed under it. In contradistinction, our algebras are not closed under any sort of involution hence they give an early indication of an asymmetry built into their very structure $a b$ initio. Indeed, in an algebraic model of dynamical quantum causal topology closely related to the one presented above, it was argued [30] that the fundamental quantum time asymmetry must be encoded already at the structural or kinematical level of the theory so that the dynamics merely 'carries',

\footnotetext{
${ }^{11}$ We wish to thank Chris Isham and Lee Smolin for pointing this out in private communication. See also 24, 30].
} 
respects or preserves this kinematical quantum arrow of time.

Fourth, it has also been widely suspected that in the quantum deep, precisely due to the potent dynamical fluctuations that the whole spacetime edifice is subject to, there should not be a sharply defined local causal structure. The local lightcone of the classical curved spacetime manifold of general relativity becomes some kind of a fuzzy structure in the sense that the classical relativistic causal connections between events should be replaced by a more general, because probabilistic, sort of connections between quantum states of spacetime events. Indeed, Finkelstein [14] in a discrete, relativistic and quantal model of spacetime structure and causality arrived at an irreducible uncertainty relation between the space and time coordinates - a relation that a fortiori points to the implausibility of talking about a clear-cut local lightcone structure in the quantum regime. Here too the algebras employed model quantum states of events and kinematical paths for possible dynamical transitions between various quantum spacetime topologies [31]. In a purely causal interpretation of these algebras [28 we still hold that it is meaningful to speak of well defined causal connections between events, albeit, both the connections and (the states of) the events themselves are inextricably entangled by coherently superposing with each other. In section 2 we show that there are quantum uncertainty relations built into our algebraic models of spacetime foam.

The fifth criticism of our approach to spacetime foam is a general, rather conceptual one and it is based on the now famous advice that Einstein gave to Heisenberg, namely, that it is the theory that determines what is observable in the sense that no theoretical entities should be declared up-front or 'postulated' to be observables [18]. The boundary between what can be observed and what cannot is delimited by the theoretical model itselft2. Since by 'theory' the physicist understands 'dynamics', and since we have not proposed one for quantum causality as yet, our a priori intuitive assumption of a dynamically variable quantum causal topology is on a par with the primitive intuition of the early Einstein long before he arrived at the concrete dynamics of general relativity that the spacetime geometry is the sole dynamical variable in the theory of gravity ( $i e$, the components of the spacetime metric $g_{\mu \nu}$ were insightfully identified with the gravitational potentials) 42]. The aforementioned advice of Einstein to let the theory select the measurable dynamical quantities greatly surprised and impressed Heisenberg [18].

Finally, our strong commitment to finite dimensional algebras and their finite dimensional IRs throughout this paper may strike odd a thinker who is aware of one of the most serious obstacles in attempts at uniting relativity and quantum theory, namely, that there are no finite dimensional unitary IRs of the non-compact orthochronous Lorentz group. At this early stage of the construction of the theory it seems more

\footnotetext{
${ }^{12}$ Interestingly enough, in Greek 'theory' (' $\theta \epsilon \omega \rho i \alpha$ ') is almost tautosemous to 'observation' (' $\left.\pi \alpha \rho \alpha \tau \eta \dot{\eta} \rho \eta \eta^{\prime}\right)$ !
} 
natural to us to sacrifice unitarity for finiteness since the former is usually perceived as a non-local conception since (in non-relativistic quantum mechanics) it conventionally involves an integration over all space(time). Also, the strong relationship between unitarity and the probabilistic (ie, positive definite metric/measure) interpretation of quantum mechanics does not seem to accord with the indefinite signature of the spacetime metric generated by a causality modelled after a partial order [34, 4], thus we are quite keen on abandoning unitarity altogether due to the more local and indefinite nature of our structures. To our knowledge, such an urge to study Hilbert spaces with an indefinite metric and their associated pseudo-unitary structure with its concomitant pseudo-probabilistic interpretation was prophetically anticipated in [17, 13]. On the other hand, another option open to us is to keep some sort of pseudo-unitarity in the theory and try to regard as 'effective symmetries' of our structures only those belonging to a finite subgroup of the Lorentz group - the transformations that are, say, 'available' to and link our finitary experimenters. For in any case, it has been seriously proposed that since a Lorentzian spacetime manifold may be regarded as a structure 'effective' only at classical scales, its (local) Lorentz symmetries should also be regarded as a macroscopic quantum effect - a quantum physically non-fundamental idiosyncracy of the classical continuum which itself is the result of a process of quantum condensation [16, 3]. The motivating insight for this proposal is that if the system (here, spacetime) is fundamentally taken to be quantum, then so must its symmetries [16]. Similarly, in 24] it has been proposed that since the differential Lorentzian manifold has been effectively 'reticularized', so must be the group manifold of its local spin-Lorentz (ie, $S L(2, \mathbf{C})$ ) gauge symmetries.

In closing this paper we would like to speculate very briefly on the future development of this approach of ours to spacetime foam. For one thing, since we have not provided an explicit dynamics for quantum causal topology, we are still haunted by Sorkin's original question: 'How does one vary a (locally finite) poset?' [40, which, in the quantum causal set context, translates to: 'How does one vary a (finite dimensional) ring? - the basic shortcoming of our scheme so far being that it provides a possible model of the kinematics of an observable quantum causality. Loosely speaking, what we know now is: 'Where does one vary a poset' - the 'kinematical rooms' in which quantum causality can possibly vary; while a concrete dynamics still remains an open theoretical question. Evidently, this then seems to be the future of our quest: find a cogent dynamical scenario for quantum causal sets. Formally, significant advances towards this end have already been made from a sheaf and topos-theoretic point of view [24, 30], but still a lot of work has to be done in order to bring together diverse ideas from the by now quite wide causal set approach to quantum gravity and amalgamate them with our algebraic approach to spacetime foam described above.

Acknowledgments. IR wishes to thank Chris Isham and Tasos Mallios for numerous exchanges on quantum causality and advice on its possible dynamics, as well as Chris 
Mulvey for helpful interactions on what 'ought to' qualify as non-commutative topology proper. He also acknowledges the financial support of the EU in the form of a Marie Curie Research Fellowship. RRZ acknowledges the 'encouraging criticism' by A.A.Grib and S.Krasnikov and the financial support from the European Research Project QACTA and from the state research program 'Universities of Russia'. In the revised version of the paper the authors express their gratitude to the referee of 'Classical and Quantum Gravity' for essential and highly constructive remarks.

\section{References}

[1] Aleksandrov, P.S., Combinatorial Topology, Greylock, Rochester, New York (1956)

[2] Baehr, H. C., Dimakis, A. and Müller-Hoissen, F., Differential calculi on commutative algebras, Journal of Physics A: Mathematical and General, 28, 3197 (1995); hep-th/9412069.

[3] Baugh, J., Finkelstein, D. R., Saller, H. and Tang, Z., General Covariance is BoseEinstein Statistics, in On Einstein's Path: Essays in Honour of Schücking, E., Ed. Harvey, A., Springer-Verlag, New York (1999).

[4] Bombelli, L., Lee, J., Meyer, D. and Sorkin, R. D., Space-Time as a Causal Set, Physical Review Letters, 59, 521 (1987).

[5] Breslav, R. B., Parfionov, G. N. and Zapatrin, R. R., Topology measurement within the histories approach, Hadronic Journal, 22, 225 (1999); quant-ph/9903011.

[6] Breslav, R. B., and Zapatrin, R. R., Differential Structure of Greechie Logics, International Journal of Theoretical Physics, 39, 1027 (2000); quant-ph/9902065.

[7] Butterfield, J. and Isham, C. J., Some Possible Roles for Topos Theory in Quantum Theory and Quantum Gravity, preprint Imperial/TP/98-99/76., to appear in a special Festschrift issue of the Foundations of Physics in honour of Marisa Dalla Chiara (2001); gr-qc/9910005.

[8] Crane, L., Clock and Category: is quantum gravity algebraic?, Journal of Mathematical Physics, 36, 6180 (1995).

[9] Eilenberg, S. and Steenrod, N., Foundations of Algebraic Topology, Princeton University Press, Princeton, New Jersey (1952)

[10] Einstein, A., Über den Äther, Schweizerische Naturforschende Gesellschaft Verhanflungen, 105, 85 (1924); English translation by Simon Saunders: On the Ether, in The Philosophy of Vacuum, Eds. Saunders, S. and Brown, H., Clarendon Press, Oxford, (1991). 
[11] Einstein, A., Physics and Reality, Journal of the Franklin Institute, 221, 313.

[12] Einstein, A., The Meaning of Relativity, Princeton University Press, Princeton (1956); 3rd extended edition (1990).

[13] Feynman, R. P., The Need for Negative Probabilities, in Quantum Implications: Essays in Honour of David Bohm, Eds. Hiley, B. J. and Peat, D., Routledge, London (1984).

[14] Finkelstein, D., Space-Time Code, Physical Review, 184, 1261 (1969).

[15] Finkelstein, D., 'Superconducting' Causal Nets, International Journal of Theoretical Physics, 27, 473 (1988).

[16] Finkelstein, D., Quantum Relativity: A Synthesis of the Ideas of Einstein and Heisenberg, Springer-Verlag, New York (1996).

[17] Heisenberg, W., Hilbert Space with Indefinite Metric, Zeitschrift für Naturforschung, 9a, 292 (1954).

[18] Heisenberg, W., Conversations with Einstein, Princeton University Press, Princeton (1996 re-print).

[19] Isham, C. J., Quantum Topology and the Quantisation on the Lattice of Topologies, Classical and Quantum Gravity, 6, 1509 (1989).

[20] Isham, C. J., An introduction to general topology and quantum topology, in Proceedings of the NATO ASI on Physics, Geometry and Topology (August, 1989), 1-64, Plenum Press (1989).

[21] Landi, G., and Lizzi, F., Projective Systems of Noncommutative Lattices as a Pregeometric Substratum, in Quantum Groups and Fundamental Physical Applications, ISI Guccia, Palermo, December 1997, D. Kastler and M. Rosso Eds., Nova Science Publishers, Inc. (1999); math-ph/9810011.

[22] Mallios, A., Geometry of Vector Sheaves: An Axiomatic Approach to Differential Geometry, vols. 1-2, Kluwer Academic Publishers, Dordrecht (1998); (volume 3 currently in press).

[23] Mallios, A., private communication

[24] Mallios, A. and Raptis, I., Finitary Spacetime Sheaves of Quantum Causal Sets: Curving Quantum Causality, International Journal of Theoretical Physics, to appear 40, (11) (2000). 
[25] Mulvey, C. and Pelletier, J. W., On the Quantization of Points, Journal of Pure and Applied Algebra, to appear (2001).

[26] Mulvey, C. and Pelletier, J. W., On the Quantization of Spaces, Journal of Pure and Applied Algebra, to appear (2001).

[27] Penrose, R., Newton, Quantum Theory and Reality, in 300 Years of Gravitation, Eds. Hawking, S. W. and Israel, W., Cambridge University Press, Cambridge (1987).

[28] Raptis, I., Algebraic Quantization of Causal Sets, International Journal of Theoretical Physics, 39, 1233 (2000).

[29] Raptis, I., Finitary Spacetime Sheaves, International Journal of Theoretical Physics, 39, 1703 (2000).

[30] Raptis, I., Non-Commutative Topology for Curved Quantum Causality, submitted to the International Journal of Theoretical Physics (2000); gr-qc/0101082.

[31] Raptis, I. and Zapatrin, R. R., Quantization of discretized spacetimes and the correspondence principle, International Journal of Theoretical Physics, 39, 1 (2000); gr-qc/9904079.

[32] Regge, T., General relativity without coordinates, Nuovo Cimento, 19, 558 (1961).

[33] Rideout, D. P. and Sorkin, R. D., A Classical Sequential Growth Dynamics for Causal Sets, Physical Review D, 61, 024002 (2000); gr-qc/9904062.

[34] Robb, A. A., Geometry of Space and Time, (1st edition), Cambridge University Press, Cambridge (1914); (2nd edition, CUP, 1936).

[35] Rota, G.-C., On The Foundation Of Combinatorial Theory, I. The Theory Of Möbius Functions, Zeitschrift für Wahrscheinlichkeitstheorie, 2, 340 (1968).

[36] Sorkin, R. D., Does a Discrete Order Underlie Spacetime and its Metric? in Proceedings of the Third Canadian Conference on General Relativity and Relativistic Astrophysics, Eds. Cooperstock, F. and Tupper, B., World Scientific, Singapore (1990).

[37] Sorkin, R. D., Spacetime and Causal Sets, in the Proceedings of the SILARG VII Conference, Cocoyoc, Mexico, preprint (1990).

[38] Sorkin, R. D., Finitary Substitute for Continuous Topology, International Journal of Theoretical Physics, 30, 923 (1991). 
[39] Sorkin, R. D., A Specimen of Theory Construction from Quantum Gravity, in The Creation of Ideas in Physics, Ed. Leplin, J., Kluwer Academic Publishers, Dordrecht (1995).

[40] Sorkin, R. D., private communication

[41] Stanley, R. P., Enumerative combinatorics, Wadsworth and Brooks, Monterey, California (1986).

[42] Torretti, R., Relativity and Geometry, Dover Publications, New York (1981).

[43] Wheeler, J. A., Geometrodynamics, in Relativity, Groups and Topology, Eds. De Witt, C. and De Witt, B. S., Gordon and Breach, London (1964).

[44] Zapatrin, R. R., Pre-Regge Calculus: Topology Via Logic, International Journal of Theoretical Physics, 32, 779 (1993).

[45] Zapatrin, R. R., Finitary Algebraic Superspace, International Journal of Theoretical Physics, 37, 799 (1998); gr-qc/9704062.

[46] Zapatrin, R. R., Incidence algebras of simplicial complexes, Pure Mathematics and its Applications, to appear (2001); math.CO/0001065.

[47] Zeeman, E. C., Causality Implies the Lorentz Group, Journal of Mathematical Physics, 5, 490 (1964). 